\title{
Inequalities related to Bourin and Heinz means with a complex parameter*
}

\author{
T. Bottazzi, R. Elencwajg, G. Larotonda and A. Varela ${ }^{\dagger}$
}

\begin{abstract}
A conjecture posed by S. Hayajneh and F. Kittaneh claims that given $A, B$ positive matrices, $0 \leq t \leq 1$, and any unitarily invariant norm it holds

$$
\left\|A^{t} B^{1-t}+B^{t} A^{1-t}\left|\|\leq\| A^{t} B^{1-t}+A^{1-t} B^{t}\right|\right\| .
$$

Recently, R. Bhatia proved the inequality for the case of the Frobenius norm and for $t \in\left[\frac{1}{4} ; \frac{3}{4}\right]$. In this paper, using complex methods we extend this result to complex values of the parameter $t=z$ in the strip $\left\{z \in \mathbb{C}: \operatorname{Re}(z) \in\left[\frac{1}{4} ; \frac{3}{4}\right]\right\}$. We give an elementary proof of the fact that equality holds for some $z$ in the strip if and only if $A$ and $B$ commute. We also show a counterexample to the general conjecture by exhibiting a pair of positive matrices such that the claim does not hold for the uniform norm. Finally, we give a counterexample for a related singular value inequality given by $s_{j}\left(A^{t} B^{1-t}+B^{t} A^{1-t}\right) \leq s_{j}(A+B)$, answering in the negative a question made by K. Audenaert and F. Kittaneh 1
\end{abstract}

\section{Introduction}

We begin this paper with some notations and definitions. The context here is the algebra of $n \times n$ complex entries matrices, but the proofs adapt well to other (infinite dimensional) settings in operator theory, so let us assume that $\mathcal{A}$ stands for an operator algebra with trace, for instance $\mathcal{A}=M_{n}(\mathbb{C})$ with its usual trace, or $\mathcal{A}=B_{2}(H)$, the Hilbert-Schmidt operators acting on a separable complex Hilbert space with the infinite trace, or $\mathcal{A}=$ $(\mathcal{A}, \operatorname{Tr})$ a $C^{*}$-algebra with a finite faithful trace.

*2000 MSC. Primary 15A45, 47A30; Secondary 15A42, 47A63.

$\dagger$ All authors supported by Instituto Argentino de Matemática, CONICET and Universidad Nacional de General Sarmiento.

${ }^{1}$ Keywords and phrases: Frobenius norm, Heinz mean, matrix inequality, matrix power, positive matrix, trace inequality, unitarily invariant norm. 
Definitions 1.1. Let $\||\cdot|\|$ denote an unitarily invariant norm on $\mathcal{A}$, which we assume is equivalent to a symmetric norm, that is

$$
\|X Y Z\|\|\leq\| X\left\|_{\infty}\right\| Y\|\mid\| Z \|_{\infty}
$$

whenever $Y \in \mathcal{A}$ (from now on $\|\cdot\|_{\infty}$ will denote the norm of the operator algebra).

For convenience we will use the notation $\tau(X)=\operatorname{Re} \operatorname{Tr}(X)$. Let $|X|=\sqrt{X^{*} X}$ stand for the modulus of the matrix or operator $X$, then the (right) polar decomposition of $X$ is given by $X=U|X|$ where $U$ is a unitary such that $U$ maps $\operatorname{Ran}|X|$ into $\operatorname{Ran}(X)$ and is the identity on $\operatorname{Ran}|X|^{\perp}=\operatorname{Ker}(X)$. Note that $\|X\|_{2}^{2}=\operatorname{Tr}\left(X^{*} X\right)=\operatorname{Tr}\left[|X|^{2}\right]$.

Consider the inequality

$$
\tau\left(A^{z} B^{z} A^{1-z} B^{1-z}\right) \leq \tau(A B),
$$

for positive invertible operators $A, B>0$ in $\mathcal{A}$, and $z \in \mathbb{C}$. We introduce some notation regarding vertical strips in the complex plane: let

$$
\mathcal{S}_{0}=\{z \in \mathbb{C}: 0 \leq \operatorname{Re}(z) \leq 1\}, \quad \mathcal{S}_{1 / 4}=\{z \in \mathbb{C}: 1 / 4 \leq \operatorname{Re}(z) \leq 3 / 4\} ;
$$

we will study the validity of (11) in both $S_{0}$ and $S_{1 / 4}$.

Intimately related to the expression above are the inequalities

$$
\left\|b _ { t } ( A , B ) \left|\||| \leq\| h_{t}(A, B)\|\|\right.\right.
$$

and

$$
\left\|b_{t}(A, B)\right\||| \leq\|A+B\| \|,
$$

for positive matrices $A, B \geq 0$ in $\mathcal{A}$, where

$$
b_{t}(A, B)=A^{t} B^{1-t}+B^{t} A^{1-t} \quad t \in[0,1]
$$

the name $b_{t}$ is due to Bourin, who conjectured inequality (3) for $n \times n$ matrices in [5], and

$$
h_{t}(A, B)=A^{t} B^{1-t}+A^{1-t} B^{t} \quad t \in[0,1]
$$

is named after Heinz, and the well-known [7] inequality

$$
\left\|\left|h_{t}(A, B)\right|\right\| \leq\||A+B \||
$$

carrying his name.

Recently, S. Hayajneh and F. Kittanneh proposed in [6] that the stronger (2) should also be valid in $M_{n}(\mathbb{C})$; however, numerical computations (see Section 31) show that, at least for the uniform norm, this is false. 
If we focus on the case $\||X|\|=\|X\|_{2}=\operatorname{Tr}\left(X^{*} X\right)^{1 / 2}$ (the Frobenius norm in the case of $n \times n$ matrices) and we write $h_{t}=h_{t}(A, B), b_{t}=b_{t}(A, B)$, then

$$
\begin{aligned}
\operatorname{Tr}\left|b_{t}\right|^{2} & =\tau\left(b_{t}^{*} b_{t}\right)=\tau\left(B^{1-t} A^{t}+A^{1-t} B^{t}\right)\left(A^{t} B^{1-t}+B^{t} A^{1-t}\right) \\
& =\tau\left(B^{2(1-t)} A^{2 t}\right)+\tau\left(A^{2(1-t)} B^{2 t}\right)+2 \tau\left(A^{t} B^{t} A^{1-t} B^{1-t}\right)
\end{aligned}
$$

where we have repeatedly used the ciclicity of $\tau$ (i.e. $\tau(X Y)=\tau(Y X))$ and the fact that $\tau\left(Z^{*}\right)=\tau(Z)$. Likewise

$$
\operatorname{Tr}\left|h_{t}\right|^{2}=\tau\left(B^{2(1-t)} A^{2 t}\right)+\tau\left(A^{2(1-t)} B^{2 t}\right)+2 \tau(A B) .
$$

Thus, proving that $\left\|b_{t}\right\|_{2} \leq\left\|h_{t}\right\|_{2}$ amounts to prove that

$$
\tau\left(A^{t} B^{t} A^{1-t} B^{1-t}\right) \leq \tau(A B),
$$

and in fact, it is clear that both inequalities are equivalent -as remarked in [6]-.

\section{Main results}

We will divide the problem in regions of the plane (or the line), and then we will also consider the possiblity of attaining the equality; we will see that this is only possible in the trivial case, i.e. when $A, B$ commute. We recall the generalized Hölder inequality, that we will use frequently: let $\frac{1}{p}+\frac{1}{q}+\frac{1}{r}=1$ for $p, q, r \geq 1$ and $X, Y, Z$ in $\mathcal{A}$, then

$$
\tau(X Y Z) \leq\|X Y Z\|_{1} \leq\|X\|_{p}\|Y\|_{q}\|Z\|_{r} .
$$

This is just a combination of the usual Hölder inequality together with

$$
\|X Y\|_{s} \leq\|X\|_{p}\|Y\|_{q}
$$

provided $s \geq 1$ and $\frac{1}{p}+\frac{1}{q}=\frac{1}{s}$ (see [8], Theorem 2.8, for more details).

\subsection{The inequality in the $\operatorname{strip} \mathcal{S}_{1 / 4}$}

We begin with an easy consequence of an inequality due to Araki-Lieb and Thirring.

Lemma 2.1. If $A, B \geq 0$ and $r \geq 2$, then

$$
\left\|A^{1 / r} B^{1 / r}\right\|_{r} \leq \tau(A B)^{1 / r} .
$$

Proof. Note that

$$
\left\|A^{1 / r} B^{1 / r}\right\|_{r}^{r}=\tau\left(\left[A^{1 / r} B^{1 / r} B^{1 / r} A^{1 / r}\right]^{r / 2}\right)=\tau\left(\left[A^{1 / r} B^{2 / r} A^{1 / r}\right]^{r / 2}\right)
$$

which, by the inequality of Araki-Lieb and Thierring (see [2], and note that $r / 2 \geq 1$ ) is less or equal than

$$
\tau\left(A^{r / 2 r} B^{r 2 / 2 r} A^{r / 2 r}\right)=\tau\left(A^{1 / 2} B A^{1 / 2}\right)
$$

which in turn equals $\tau(A B)$. 
Note that if we exchange the variables $z \mapsto 1-z$ and exchange the role of $A, B$, it suffices to consider half-strips or half-intervals around $\operatorname{Re}(z)=1 / 2$.

Proposition 2.2. If $0<A, B$ and $z \in \mathcal{S}_{1 / 4}$, then

$$
\tau\left(A^{z} B^{z} A^{1-z} B^{1-z}\right) \leq \tau(A B) .
$$

Proof. Let $z=1 / 2+i y, y \in \mathbb{R}$ denote any point in vertical line of the complex plane passing through $x=1 / 2$. Then

$$
\begin{aligned}
\tau\left(A^{z} B^{z} A^{1-z} B^{1-z}\right) & =\tau\left(A^{i y} A^{1 / 2} B^{1 / 2} B^{i y} A^{-i y} A^{1 / 2} B^{1 / 2} B^{-i y}\right) \\
& \leq \tau\left|A^{i y} A^{1 / 2} B^{1 / 2} B^{i y} A^{-i y} A^{1 / 2} B^{1 / 2} B^{-i y}\right| \\
& \leq\left\|A^{i y} A^{1 / 2} B^{1 / 2} B^{i y} A^{-i y}\right\|_{2}\left\|A^{1 / 2} B^{1 / 2} B^{-i y}\right\|_{2}=\left\|A^{1 / 2} B^{1 / 2}\right\|_{2}^{2}
\end{aligned}
$$

by the Cauchy-Schwarz inequality and the fact that $A^{i y}, B^{i y}$ are unitary operators. Then by the previous lemma,

$$
\tau\left(A^{z} B^{z} A^{1-z} B^{1-z}\right) \leq \tau(A B)^{2 / 2}=\tau(A B) .
$$

Now consider $z=1 / 4+i y, y \in \mathbb{R}$, a generic point in the vertical line over $x=1 / 4$, then noting that $\frac{1}{4}+\frac{1}{4}+\frac{1}{2}=1$,

$$
\begin{aligned}
\tau\left(A^{z} B^{z} A^{1-z} B^{1-z}\right) & =\tau\left(B^{1 / 4} A^{1 / 4} A^{i y} B^{i y} B^{1 / 4} A^{1 / 4} A^{-i y} A^{1 / 2} B^{1 / 2} B^{-i y}\right) \\
& \leq\left\|B^{1 / 4} A^{1 / 4}\right\|_{4}^{2}\left\|B^{1 / 2} A^{1 / 2}\right\|_{2} \leq \tau(A B)^{2 / 4+1 / 2}=\tau(A B),
\end{aligned}
$$

where we used again the previous Lemma and the generalized Hölder's inequality,

$$
\tau(X Y Z) \leq\|X\|_{p}\|Y\|_{q}\|Z\|_{r}
$$

whenever $p, q, r \geq 1$ and $\frac{1}{p}+\frac{1}{q}+\frac{1}{r}=1$.

By Hadamard's three-lines theorem, the bound $\tau(A B)$ is valid in the vertical strip $1 / 4 \leq$ $\operatorname{Re}(z) \leq 1 / 2$, since it holds in the frontier of the strip. Invoking the symmetry $z \mapsto 1-z$ and exchanging the roles of $A, B$ gives the desired bound on the full strip $\mathcal{S}_{1 / 4}=\{1 / 4 \leq$ $\operatorname{Re}(z) \leq 3 / 4\}$

Regarding the inequalities conjectured by Bourin et al., note that we can assume $A, B>0$ : replacing $A$ with $A_{\varepsilon}=A+\varepsilon$ (and likewise with $B$ ), if the inequality (1) is valid for $A_{\varepsilon}, B_{\varepsilon}$ then making $\varepsilon \rightarrow 0^{+}$gives the general result: the following result that we state as corollary was recently obtained by R. Bhatia in [4] and we should also point the reader to the paper by T. Ando, F. Hiai, K. Okubo [1].

Corollary 2.3. For any $A, B \geq 0$ and any $t \in[1 / 4,3 / 4]$,

$$
\left\|A^{t} B^{1-t}+B^{t} A^{1-t}\right\|_{2} \leq\left\|A^{t} B^{1-t}+A^{1-t} B^{t}\right\|_{2} \leq\|A+B\|_{2} .
$$




\subsection{Inequality becomes equality}

Let us consider the special case when the inequality above becomes an equality. We begin with a lemma that we will use in several ocasions, and will be useful when we drop the assumption on nonsingularity of $A, B$.

Lemma 2.4. Let $A, B \geq 0$, and assume

$$
\tau\left(A^{1 / 2} B^{1 / 2} A^{1 / 2} B^{1 / 2}\right)=\tau(A B)
$$

or

$$
\left\|A^{1 / 4} B^{1 / 4}\right\|_{4}=\tau(A B)^{1 / 4} .
$$

In either case, $A$ commutes with $B$.

Proof. Name $X=A^{1 / 2} B^{1 / 2}$, and considering the inner product induced by $\tau,\langle X, Y\rangle=$ $\tau\left(X Y^{*}\right)$

$$
\left\langle X, X^{*}\right\rangle=\tau\left(X^{2}\right)=\tau\left(A^{1 / 2} B^{1 / 2} A^{1 / 2} B^{1 / 2}\right)=\tau(A B)=\tau\left(X^{*} X\right)=\|X\|_{2}^{2}=\|X\|_{2}\left\|X^{*}\right\|_{2} .
$$

But Cauchy-Schwarz inequality becomes an equality if and only if $X=\lambda X^{*}$ for some $\lambda>0$, and since both operators have equal norm $\left(=\left\|A^{1 / 2} B^{1 / 2}\right\|_{2}\right)$, then $X=X^{*}$. This means

$$
A^{1 / 2} B^{1 / 2}=B^{1 / 2} A^{1 / 2},
$$

and this implies that $A$ commutes with $B$. On the other hand,

$$
\left\|A^{1 / 4} B^{1 / 4}\right\|_{4}^{4}=\tau\left(\left(B^{1 / 4} A^{1 / 2} B^{1 / 4}\right)^{2}\right)=\tau\left(A^{1 / 2} B^{1 / 2} A^{1 / 2} B^{1 / 2}\right),
$$

so what we have is just another way of writing the first equality condition.

Proposition 2.5. Let $A, B>0$ and assume that there is $z_{0} \in \mathcal{S}_{1 / 4}$ such that

$$
\tau\left(A^{z_{0}} B^{z_{0}} A^{1-z_{0}} B^{1-z_{0}}\right)=\tau(A B) .
$$

Then $A$ commutes with $B$ and $\tau\left(A^{z} B^{z} A^{1-z} B^{1-z}\right)=\tau(A B)$ for any $z \in \mathbb{C}$.

Proof. First consider the case when equality is reached in an interior point of the strip $\mathcal{S}_{1 / 4}$. Note that by the maximum modulus principle, this would mean that the function

$$
f(z)=\tau\left(A^{z} B^{z} A^{1-z} B^{1-z}\right)
$$

is constant in the strip $\mathcal{S}_{1 / 4}$, in particular equality holds at $z_{0}=1 / 2$, and by the previous Lemma, $A$ commutes with $B$. 
Now suppose equality is attained in the frontier, for instance at $z_{0}=1 / 4+i y$ for some $y \in \mathbb{R}$. Let $X=B^{1 / 4} A^{1 / 4} A^{i y} B^{i y} B^{1 / 4} A^{1 / 4}, Y=B^{1 / 2} B^{i y} A^{i y} A^{1 / 2}$. Then, if we go through the proof of Proposition 2.2 again, assuming equality

$$
\begin{aligned}
\tau(A B) & =\tau\left(X Y^{*}\right)=\langle X, Y\rangle \leq\|X\|_{2}\|Y\|_{2} \\
& \leq\left\|B^{1 / 4} A^{1 / 4}\right\|_{4}^{2}\left\|A^{1 / 2} B^{1 / 2}\right\|_{2} \leq \tau(A B) .
\end{aligned}
$$

Arguing as in the previous Lemma, there exists $\lambda>0$ such that $X=\lambda Y$,

$$
B^{1 / 4} A^{1 / 4} A^{i y} B^{i y} B^{1 / 4} A^{1 / 4}=\lambda B^{1 / 2} B^{i y} A^{i y} A^{1 / 2} .
$$

Cancelling $B^{1 / 4}$ on the left and $A^{1 / 4}$ on the right we obtain

$$
A^{1 / 4} A^{i y} B^{i y} B^{1 / 4}=\lambda B^{1 / 4} B^{i y} A^{i y} A^{1 / 4},
$$

but now both elements have the same norm and this shows that $\lambda=1$; then

$$
A^{1 / 4+i y} B^{1 / 4+i y}=B^{1 / 4+i y} A^{1 / 4+i y},
$$

and since $A, B>0$, the existence of analytic logarithms shows that again $A$ commutes with $B$. By symmetry, the same argument applies for any $z_{0}=3 / 4+i y$ in the other border of the strip.

Corollary 2.6. If $A$ does not commute with $B$, the inequality is strict:

$$
\tau\left(A^{z} B^{t} A^{1-z} B^{1-z}\right)<\tau(A B),
$$

in some open set $\Omega \subset \mathbb{C}$ containing the closed strip $\mathcal{S}_{1 / 4}$.

If we allow $A, B$ to be non invertible, holomorphy is lost, but nevertheless in the same spirit we have the following result.

Proposition 2.7. For given $A, B \geq 0$, there exists $\delta=\delta(A, B)>0$ such that

$$
\tau\left(A^{t} B^{t} A^{1-t} B^{1-t}\right) \leq \tau(A B)
$$

holds in the interval $[1 / 4-\delta, 3 / 4+\delta]$. If $A$ does not commute with $B$, the inequality is strict in the whole $(1 / 4-\delta, 3 / 4+\delta)$.

Proof. If $A$ commutes with $B$, then the assertion is trivial. If not, arguing as in the last part of the proof of the previous proposition, we must have strict inequality

$$
\tau\left(A^{t} B^{t} A^{1-t} B^{1-t}\right)<\tau(A B)
$$

for $t=1 / 4, t=3 / 4$, and then by continuity the inequality extends a bit out of the closed interval $[1 / 4,3 / 4]$. 
Consider $t \in(1 / 4,1 / 2)$ and put $X=B^{1 / 4} A^{1 / 4} A^{t-1 / 4} B^{t-1 / 4}, Y=B^{1 / 4} A^{1 / 4} A^{3 / 4-t} B^{3 / 4-t}$. Note that $\frac{1}{t}, \frac{1}{1-t} \geq 1$ and define $1 / p=t-1 / 4 \in(0,1 / 4), 1 / q=3 / 4-t \in(1 / 4,1 / 2)$, note also that $1 / p+1 / 4=t, 1 / q+1 / 4=1-t$. By reiterated use of Hölder's inequality compute

$$
\begin{aligned}
\tau\left(A^{t} B^{t} A^{1-t} B^{1-t}\right) & \leq\|X Y\|_{1} \leq\|X\|_{t^{-1}}\|Y\|_{(1-t)^{-1}} \\
& \leq\left\|B^{1 / 4} A^{1 / 4}\right\|_{4}\left\|A^{1 / p} B^{1 / p}\right\|_{p}\left\|B^{1 / q} A^{1 / q}\right\|_{q}\left\|A^{1 / 4} B^{1 / 4}\right\|_{4} .
\end{aligned}
$$

Now apply Lemma 2.1 to each of the four terms (note that $p>4$ and $q>2$ ), and we have 2

$$
\tau\left(A^{t} B^{t} A^{1-t} B^{1-t}\right) \leq\left\|B^{1 / 4} A^{1 / 4}\right\|_{4}\left\|A^{1 / p} B^{1 / p}\right\|_{p}\left\|B^{1 / q} A^{1 / q}\right\|_{q}\left\|A^{1 / 4} B^{1 / 4}\right\|_{4} \leq \tau(A B) .
$$

If we assume equality of the traces, then

$$
\tau(A B)=\left\|B^{1 / 4} A^{1 / 4}\right\|_{4}\left\|A^{1 / p} B^{1 / p}\right\|_{p}\left\|B^{1 / q} A^{1 / q}\right\|_{q}\left\|A^{1 / 4} B^{1 / 4}\right\|_{4}
$$

and in particular, it must be that $\left\|A^{1 / 4} B^{1 / 4}\right\|_{4}=\tau(A B)^{1 / 4}$, and from Lemma 2.4 we can deduce that $A$ commutes with $B$. By the symmetry $(t \mapsto 1-t)$ the argument extends to $(1 / 2,3 / 4)$, and again by Lemma 2.4 we already know that $A$ commutes with $B$ if equality is attained at $t=1 / 2$. This finishes the proof of the assertion that the inequality is strict in $[1 / 4,3 / 4]$ unless $A$ commutes with $B$.

Remark 2.8. The inequalities in the previous proof give in fact

$$
\tau\left(\left|B^{\frac{1}{4}} A^{t} B^{t} A^{1-t} B^{\frac{3}{4}-t}\right|\right) \leq \operatorname{Tr}(A B)
$$

for any $t \in\left[\frac{1}{4}, \frac{3}{4}\right]$; this is a particular instance of [1, Theorem 2.10].

\section{Counterexamples}

In this section we exhibit specific cases of different kind. In Example 3.1 we choose $A, B$ such that $\left\|b_{t}(A, B)\right\|_{\infty}>\left\|h_{t}(A, B)\right\|_{\infty}$, while in Example 3.2, it is shown that the $j^{\text {th }}$ singular value of $A+B$ is not always greater than the $j^{\text {th }}$ singular value of $b_{t}(A, B)$. This provides negative answers to [6, Conjecture 1.2] and [3, Problem 4] respectively.

Example 3.1. Consider the following positive definite matrices

$$
A=\left(\begin{array}{ccc}
1141 & 0 & 0 \\
0 & 204 & 0 \\
0 & 0 & 1 / 8
\end{array}\right) \quad \text { and } \quad B=\left(\begin{array}{ccc}
39 & 90 & 43 \\
90 & 418 & 370 \\
43 & 370 & 426
\end{array}\right)
$$

\footnotetext{
${ }^{2}$ Note that this is another proof of the inequality for real $t \in\left[\frac{1}{4}, \frac{3}{4}\right]$.
} 
The following is the graph of $f(t)=-\left\|b_{t}(A, B)\right\|_{\infty}+\left\|h_{t}(A, B)\right\|_{\infty}$ for $t \in\left[0, \frac{1}{2}\right]$ :

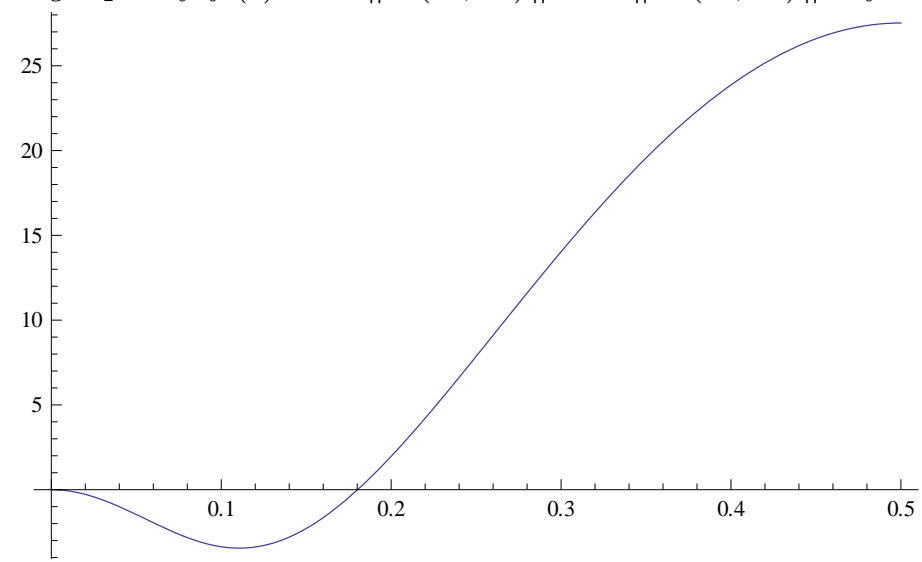

For these matrices $-\left\|b_{t}(A, B)\right\|_{\infty}+\left\|h_{t}(A, B)\right\|_{\infty} \simeq-2.3$ at $t=.15$.

In [3, Problem 4] K. Audenaert and F. Kittaneh asked if $s_{j}\left(b_{t}(A, B)\right) \leq s_{j}(A+B)$ for every $j$ and $0<t<1$ (where $s_{j}(M), j=1 \ldots n$ denote the singular values of the matrix $M$ arranged in non-increasing order).

Example 3.2. Consider the following positive definite matrices

$$
A=\left(\begin{array}{ccc}
6317 & 0 & 0 \\
0 & 474 & 0 \\
0 & 0 & 6
\end{array}\right) \quad \text { and } \quad B=\left(\begin{array}{ccc}
2078 & 2362 & 2199 \\
2362 & 3267 & 2585 \\
2199 & 2585 & 2492
\end{array}\right)
$$

Then, for $t=\frac{1}{2}$ we have

$$
s\left(b_{\frac{1}{2}}(A, B)\right)=(6826.57,878.499,591.716)
$$

and

$$
s(A+B)=(10561.4,3629.62,443.017) .
$$

In particular, $s_{3}\left(b_{\frac{1}{2}}(A, B)\right)>s_{3}(A+B)$.

\section{References}

[1] T. Ando, F. Hiai, K. Okubo. Trace inequalities for multiple products of two matrices. Math. Inequal. Appl. 3 (2000), no. 3, 307-318.

[2] H. Araki. On an inequality of Lieb and Thirring, Lett. Math. Phys. 19 (1990), pp. 167-170.

[3] K. Audenaert, F. Kittaneh. Problems and Conjectures in Matrix and Operator Inequalities, eprint arXiv:1201.5232v3 [math.FA] 
[4] R. Bhatia. Trace inequalities for products of positive definite matrices, J. Math. Phys. 55 (2014).

[5] J.C. Bourin. Matrix subadditivity inequalities and block-matrices. Internat. J. Math. 20 (2009), no. 6, 679-691.

[6] S. Hayajneh, F. Kittaneh. Lieb-Thirring trace inequalities and a question of Bourin. J. Math. Phys. 54 (2013), no. 3, 033504, 8 pp.

[7] E. Heinz. Beiträge zur Störungstheorie der Spektralzerlegung. (German) Math. Ann. 123, (1951). 415-438.

[8] B. Simon. Trace ideals and their applications. Second edition. Mathematical Surveys and Monographs, 120. American Mathematical Society, Providence, RI, 2005. 